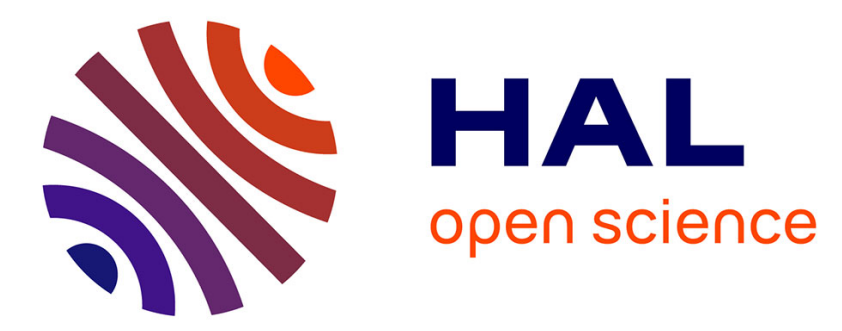

\title{
Elastic coupling between layers in two-dimensional materials
}

Yang Gao, Suenne Kim, Si Zhou, Hsiang-Chih Chiu, Daniel Nelias, Claire Berger, Walt de Heer, Laura Polloni, Roman Sordan, Angelo Bongiorno, et al.

\section{- To cite this version:}

Yang Gao, Suenne Kim, Si Zhou, Hsiang-Chih Chiu, Daniel Nelias, et al.. Elastic coupling between layers in two-dimensional materials. Nature Materials, 2015, 14 (7), pp.714 - 720. 10.1038/nmat4322 . hal-01784922

\section{HAL Id: hal-01784922 \\ https://hal.science/hal-01784922}

Submitted on 3 May 2018

HAL is a multi-disciplinary open access archive for the deposit and dissemination of scientific research documents, whether they are published or not. The documents may come from teaching and research institutions in France or abroad, or from public or private research centers.
L'archive ouverte pluridisciplinaire HAL, est destinée au dépôt et à la diffusion de documents scientifiques de niveau recherche, publiés ou non, émanant des établissements d'enseignement et de recherche français ou étrangers, des laboratoires publics ou privés. 


\title{
Elastic coupling between layers in two-dimensional materials
}

\author{
Yang Gao ${ }^{1,2}$, Suenne Kim ${ }^{3}$, Si Zhou', Hsiang-Chih Chiu ${ }^{4}$, Daniel Nélias ${ }^{5}$, Claire Berger ${ }^{1,6}$, \\ Walt de Heer ${ }^{1,7}$, Laura Polloni ${ }^{8}$, Roman Sordan ${ }^{8}$, Angelo Bongiorno ${ }^{1,9}$, Elisa Riedo ${ }^{1,2}$
}

\author{
${ }^{1}$ School of Physics, Georgia Institute of Technology, 837 State Street, Atlanta, Georgia 30332-0430, USA. \\ ${ }^{2}$ Advanced Science Research Center and City College New York, City University of New York, 85 St Nicholas Terrace, \\ New York, New York 10031, USA. \\ ${ }^{3}$ Department of Applied Physics, Hanyang University, Ansan 426-791, South Korea. \\ ${ }^{4}$ Department of Physics, National Taiwan Normal University, 88, Sec.4, Ting-Chou Road, Taipei 116, Taiwan. \\ 5 Université de Lyon, CNRS, INSA-Lyon, LaMCoS UMR5259, Villeurbanne F69621, France. \\ ${ }^{6}$ Institut Néel, Université Grenoble Alpes-CNRS, BP 166, 38042 Grenoble, France. \\ ${ }^{7}$ King Abdulaziz University, Department of Physics, Jeddah 21589, Saudi Arabia. \\ 8 L-NESS, Department of Physics, Politecnico di Milano, Via Anzani 42, 22100 Como, Italy. \\ ${ }^{9}$ Department of Chemistry, College of Staten Island, City University of New York, New York, New York 10314, USA.
}

\begin{abstract}
Two-dimensional materials, such as graphene and $\operatorname{MoS}_{2}$, are films of a few atomic layers in thickness with strong in-plane bonds and weak interactions between the layers. The in-plane elasticity has been widely studied in bending experiments where a suspended film is deformed substantially; however, little is known about the films' elastic modulus perpendicular to the planes, as the measurement of the out-of-plane elasticity of supported 2D films requires indentation depths smaller than the films' interlayer distance. Here, we report on sub-ångström-resolution indentation measurements of the perpendicularto-the-plane elasticity of 2D materials. Our indentation data, combined with semi-analytical models and density functional theory, are then used to study the perpendicular elasticity of few-layer-thick graphene and graphene oxide films. We find that the perpendicular Young's modulus of graphene oxide films reaches a maximum when one complete water layer is intercalated between the graphitic planes. This non-destructive methodology can map interlayer coupling and intercalation in 2D films.
\end{abstract}

A 1 arge s cientific and t echnological effort i s underway to understand and control the properties of $2 \mathrm{D}$ naterials because of their potential technological applications ${ }^{1-7}$. The

most studied 2D material is graphene, existing as a single layer of graphite $^{8}$ or a few-layer-thick epitaxial graphene (EG) film? ${ }^{9}$.

Graphene possesses a large in-plane Young's modulus ${ }^{10}(\sim 1 \mathrm{TPa})$ as well as high intrinsic carrier mobility $y^{11,12}$ and high in-plane thermal conductivity $^{13}$. Besides graphene, also 2D films of graphene oxide ${ }^{14-}$ ${ }^{17}(\mathrm{GO})$, hexagonal boron nitride ${ }^{18}$ and transition metal dichalcogenides ${ }^{19-21}$ such as $\mathrm{MoS}_{2}$ exhibit unique and excellent properties and hold great promise for nanotechnology applications. One of the main characteristics of $2 \mathrm{D}$ materials is the high anisotropy between the in-plane and perpendicular-to-the-plane properties. For example, in graphite, owing to the strong covalent bonds between atoms in the plane, and the weak Van der Waals interlayer interaction, the in-plane Young's modulus is $E_{\|}=1 \mathrm{TPa}$ (ref. 22), whereas the interlayer perpendicular-to-the-plane Young's modulus is only $E_{\perp}=36 \mathrm{GPa}$ (ref. 22). Recent studies have suggested that the mechanical properties of $2 \mathrm{D}$ materials are strongly correlated to their structure and properties ${ }^{1,10,23-25}$. The inplane Young's modulu ${ }^{1,10,25-27}$ of exfoliated graphene and $\mathrm{MoS}_{2}$ has been widely studied in bending experiments where a film is suspended on trenches or holes, and an atomic force microscopy (AFM) tip is used to bend the suspended film with deformations of tens and hundreds of nanometres. On the other hand, very little is known about the elasticity perpendicular to the planes, hereafter called perpendicular or interlayer elasticity, of $2 \mathrm{D}$ materials composed of very few atomic layers. Recent calculations have investigated the out-of-plane shear and Young's modulus of carbon nanotubes and graphene ${ }^{28}$. Experimentally, resonance ultrasound spectroscopy was used to study the elastic constants and the anisotropy between the in-plane and perpendicular-to-the-plane directions of thin films $\mathrm{s}^{29}$. Investigations of the perpendicular-to-the-plane elasticity of fewlayer-thick 2D films have not been reported to the best of our knowledge, and remain an experimental challenge because they require indentations on supported-as opposed to suspended-2D films, where the indentation should remain smaller than the films' interlayer distance, that is, less than a few ångströms. Nevertheless, the interlayer elastic coupling is particularly important because it is related to the thermal ${ }^{30}$, electronic ${ }^{31}$, tribological $^{24,32}$ and optical ${ }^{33}$ properties of $2 \mathrm{D}$ films. The perpendicular elasticity is expected to be affected by the structure and chemistry of the layers, the presence of stacking and intrinsic defects, and intercalation, which is a critical process for doping and tuning mechanical and electronic properties in 2D films. Mapping the interlayer elastic coupling in $2 \mathrm{D}$ films is therefore an important technological and scientific advancement.

\section{Modulated nanoindentation experiments}

Here, an unconventional AFM-based method allowing for subångström-resolution indentations coupled with a semi-analytical method (SAM) is used to measure the elasticity of 2D films in the direction perpendicular to the layers, for a number of layers as small as two. This AFM-based method is capable of maintaining an indentation depth smaller than the interlayer distance, with a resolution of $0.1 \AA$. We report on AFM-SAM investigations of the perpendicular elastic modulus of EG, epitaxial graphene oxide (EGO), and conventional GO films at varying ambient humidity. These studies, together with density functional theory (DFT) calculations, highlight how the interlayer elastic coupling in graphene and GO films is affected by the films' chemistry, structure, water intercalation, and number of layers. Interestingly, the perpendicular Young's modulus of GO increases by increasing 


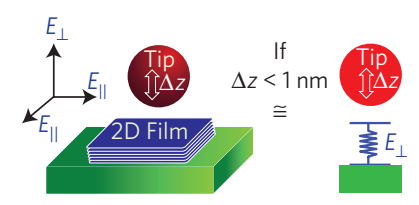

b

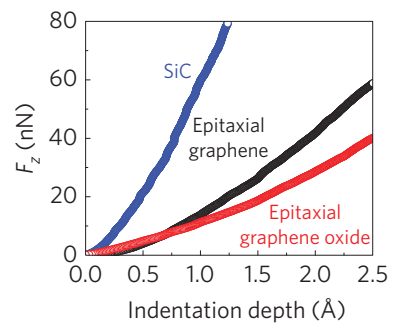

Figure 1. Modulated nanoindentation experiments. a, Schematic diagram of the experimental set-up, where a spherical AFM tip vibrates while indenting a few-layer-thick film of graphene or GO. b, Experimentally measured indentation curves for single-crystal $\mathrm{SiC}, 10$-layer-thick EG, and 10-layer-thick EGO. All three curves were obtained with the same AFM tip, $R=114 \mathrm{~nm}$.

the amount of water trapped in between the layers until a full monolayer is produced. Then, when the second water layer is forming, the modulus decreases. Finally, our studies show that when using sub-interlayer-distance indentations in 2D films thicker than a few layers, the AFM indentation curves are very sensitive to the elastic modulus perpendicular to the layers, $E_{\perp}$, and almost independent of the value of the in-plane Young's modulus, $E_{\|}$(see Fig. 1a). As a consequence, the indentation force curves can be fitted with a simple modified Hertz model as if the film is an isotropic material, where Young's modulus of the film is indeed the perpendicular modulus $E_{\perp}$. This work is therefore offering a new experimental and theoretical framework to investigate the interlayer elastic coupling in 2D films.

The experimental measurement of the perpendicular-to-theplane elasticity of 2D films is here achieved by using an unconventional AFM-based method that we call modulated nanoindentation ${ }^{14,34,35}(\mathrm{MoNI})$. In particular, we have investigated highly oriented pyrolytic graphite (HOPG), 4H-SiC, EG films grown on a $\mathrm{SiC}$ substrate ${ }^{2,9}, \mathrm{EGO}$ films grown on a $\mathrm{SiC}$ substrate ${ }^{36}$, and regular GO films deposited with the conventional Hummers' method ${ }^{37}$ on a Si substrate. More details about indentation experiments, sample preparation and properties of the films are provided in Methods and Supplementary Information (see also Supplementary Figs 1-5). During a typical MoNI experiment, an AFM tip, which is vertically oscillated at a fixed frequency with a $\sim 0.1 \AA$ amplitude, applies an increasing pressure to a 2D film surface, in the $z$-direction perpendicular to the film surface (see Fig. 1a). The oscillations are applied and controlled by a lock-in amplifier, while a constant normal force $F_{z}$ between the tip and the 2D film is maintained constant by the feedback loop of the AFM. By working with a constant force, any thermal drift is avoided. Furthermore, using a lock-in detection system together with a differential measurement allows us to measure very shallow indentations, usually smaller than $1-3 \AA$, with a resolution of $0.1 \AA$. Indeed, instead of measuring directly the normal force as a function of the indentation, $z_{\text {indent, }}$ we measure the slope of the force versus indentation curve at each constant normal force, namely $k_{\text {cont }}\left(F_{z}\right)$. Force versus indentation curves are then obtained by integrating the equation $\mathrm{d} F_{z}=k_{\text {cont }}\left(F_{z}\right) \cdot \mathrm{d} z_{\text {indent }}$ as follows:

$$
z_{\text {indent }}\left(F_{z}\right)=\int_{0}^{F_{z}} \frac{\mathrm{d} F_{z}}{k_{\text {cont }}\left(F_{z}\right)}
$$

This experimental set-up is therefore uniquely suited to investigate the perpendicular elastic modulus of $2 \mathrm{D}$ films, which are only a few layers thick. We note that the lower limit of the integral in equation (1) is zero only when there are no adhesion forces. In the case of an adhesive contact, the lower limit is the pull-off force, $F_{\mathrm{po}}$, defined as the negative load at which the AFM tip loses the contact with the sample and can be easily determined experimentally (Supplementary Figs 2 and 3 for more details) ${ }^{38-40}$. Furthermore, for $\mathrm{SiC}$ and the $2 \mathrm{D}$ materials investigated here, when the load is equal to $F_{\mathrm{po}}$ the contact area is indeed zero and $z_{\text {indent }}\left(F_{\mathrm{po}}\right)=0$ (see Supplementary Information). As shown in Supplementary Fig. 3, $k_{\text {cont }}\left(F_{z}\right)$-which is proportional to the contact radius and the squared root of the indentation depth-drops to zero when the load reaches $F_{\text {po }}$. We underline that the $F_{z}$ versus $z_{\text {indent }}$ curves could in principle be shifted along the $z_{\text {indent }}$ axis in very soft and adhesive materials depending on the value of $z_{\text {indent }}\left(F_{\mathrm{po}}\right)$; however, the shape of these curves does not depend on the type of adhesive contact. We also note that in Supplementary Figs 2 and 3 the pull-off force can change significantly from curve to curve; however, $\Delta F_{z}$ remains consistent. This shift in the absolute value of the normal force is due to humidity and the drifting of the laser position on the photo-detector during the different individual experiments (we take hundreds of curves for each sample and condition). More detailed discussions can be found in the Supplementary Information and Supplementary Figs 4 and 5. However, the clear determination of the pull-off force during each indentation experiment allows one to always determine the corrected 'absolute' normal load as $F_{z}=\left(F_{z}-F_{\mathrm{po}}\right)$, and to compare different curves independently of the laser position shifting and presence of adhesion forces. In the Supplementary Information we discuss in detail why for the materials investigated here we can account for the adhesion force by simply adding to the load the pull-off force. This adhesive contact mechanics model is called the DerjaguinMuller-Toporov (DMT) model ${ }^{40,41}$ and it describes well stiff materials and small tip radii. Figure $1 \mathrm{~b}$ shows the measured (and offset-corrected) $F_{z}$ versus $z_{\text {indent }}$ at ambient humidity for different 2D films, namely EG and EGO. We remark that both EG and EGO in Fig. $1 \mathrm{~b}$ are 10 layers thick. Figure $1 \mathrm{~b}$ also shows the force versus indentation curve for a single crystal of silicon carbide, the substrate over which EG and EGO are grown. The indentation curves, all performed with the same AFM tip, clearly indicate a larger stiffness for 10-layer EG than 10-layer EGO. As expected from previous studies, $\mathrm{SiC}$ shows a very large stiffness. $4 \mathrm{H}-\mathrm{SiC}$ has a hexagonal crystal structure; however, its elastic behaviour is very isotropic, and therefore a simple Hertzian

offset-corrected contact model can be used to extract from the force versus indentation curves Young's modulus of the material. For the configuration of a sphere (the AFM tip) pressing on a flat surface of an isotropic half-space with a normal force $F_{z}$ the Hertz model gives:

$$
F_{z}=\frac{4}{3} E^{*}(R)^{1 / 2} z_{\text {indent }}^{3 / 2}
$$

where $E^{*}=\left(\left(1-\left(v_{\text {Hertz }}^{\text {sample }}\right)^{2} / E_{\text {Hertz }}^{\text {sample }}\right)+\left(1-\left(v_{\text {Hertz }}^{\text {tip }}\right)^{2} / E_{\text {Hertz }}^{\text {tip }}\right)\right)$, with $v_{\text {Hertz }}^{\text {sampletip }}$ and $E_{\text {Hertz }}^{\text {sampletip }}$ being Poisson's ratio and Young's moduli of, respectively, the investigated sample and the AFM tip, with $R$ the AFM-tip radius $^{42}$. For the silicon tip used in the MoNI measurements, $E_{\text {Hertz }}^{\text {tip }}=169 \mathrm{GPa}$ and $v_{\text {Hertz }}^{\text {tip }}=0.27$. The elastic modulus can therefore be obtained by using the relationship (2) to fit the experimental measurements of $F_{z}$ versus $z_{\text {indent }}$, as shown in Fig. $1 \mathrm{~b}$ for a quasi-isotropic sample such as $\mathrm{SiC}$. Indeed, the fitting procedure gives $E_{\mathrm{Hertz}}^{\mathrm{SiC}}=400 \mathrm{GPa}$, for a tip radius $R=114 \mathrm{~nm}$ (the details of tip radius measurement can be found in the Supplementary Information and Supplementary Fig. 6), which is in excellent agreement with the literature. Similar experiments have also been performed on other standard samples such as $\mathrm{ZnO}$ single crystals to ensure the ability of MoNI to obtain reliable results (see details in the Supplementary Information and Supplementary Fig. 2). However, the Hertz relationship in (2) is in principle not well suited to model the contact mechanics between an AFM spherical tip and an anisotropic material such as graphite and other $2 \mathrm{D}$ materials because the Hertz model was originally valid only for isotropic half-spaces ${ }^{42,43}$. 
a

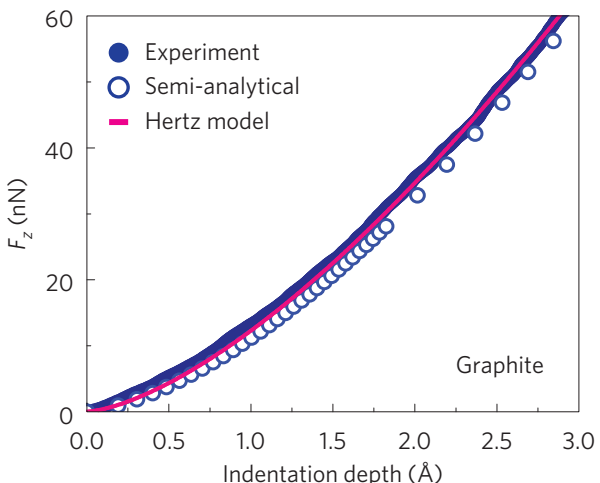

c

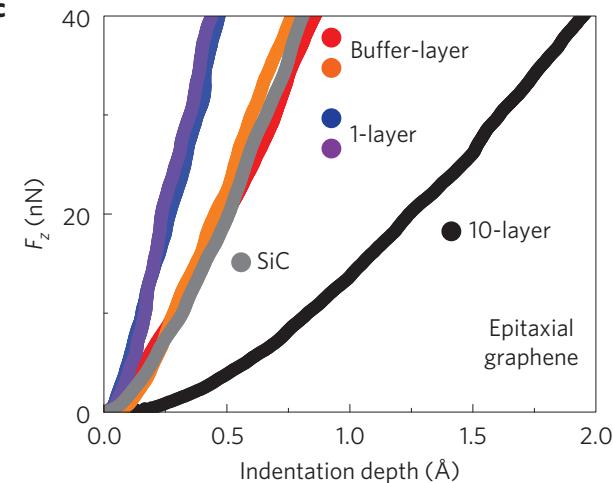

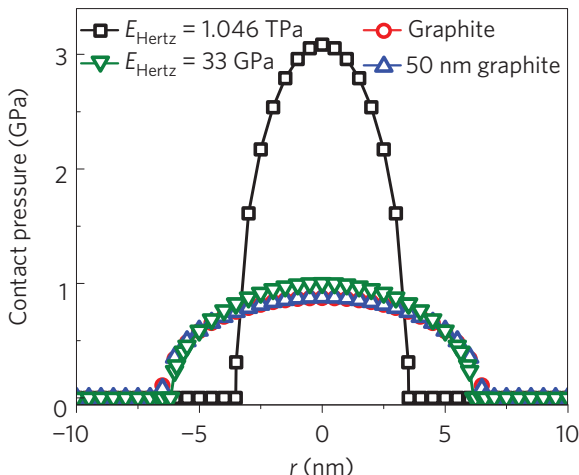

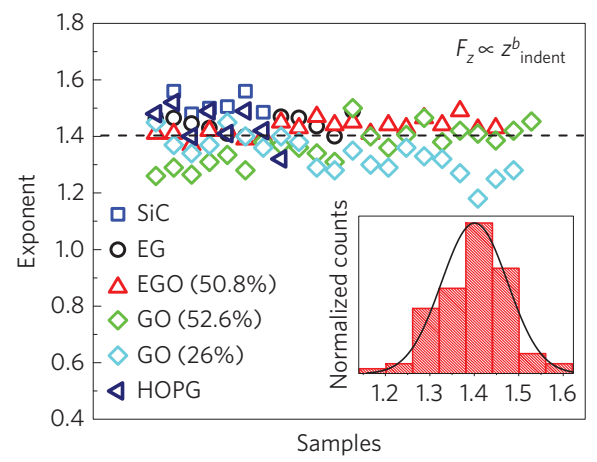

Figure 2. Experimental, SAM-simulated and Hertz indentation curves. a, Experimentally measured indentation curves in HOPG (filled circles), semi-analytical model simulations of indentation in graphite (open circles), and Hertzian fitting (continuum line) of the indentation curves on HOPG. The indenting tip radius was $100 \mathrm{~nm}$. b. Contact-pressure distribution profiles for Hertz contacts and SAM simulations of indentation in graphite. Note that for bulk graphite and for a graphite film $50 \mathrm{~nm}$ thick, the SAM simulations and the contact distribution profiles almost overlap. c, Experimental indentation curves on 10-layer-thick EG, 1-layer-thick EG, buffer-layer EG, and SiC. $\mathbf{d}$, Statistical analysis of exponent number $b$ in the fitting function $F_{z}=C^{*} z_{\text {indent }}^{b}$. For $\mathrm{EGO}$ and $\mathrm{GO}$, the $\mathrm{RH}$ is indicated. $b_{\text {average }}$ is 1.40 .

\section{Semi-analytical methods}

A better insight into the contact-pressure distribution as a function of the material elastic constants can be obtained using simulations with SAMs, which have proved their efficiency in describing the contact mechanics of anisotropic materials ${ }^{44-46}$. Here, we use SAM to simulate the force versus indentation curves in graphite. We use graphite elastic constants found in the literature 22 (see Methods), and we model the indentation of an AFM silicon tip $(R=100 \mathrm{~nm})$ in a graphite sample deposited on $\mathrm{SiC}$. We use this configuration because the $2 \mathrm{D}$ materials studied here have been deposited either on $\mathrm{SiC}$ (EG and EGO) or Si (conventional GO). Figure 2a shows the results from the SAM simulations on graphite along with the experimental curves obtained by MoNI on a bulk sample of HOPG. In Fig. 2a, the SAM-simulated curve agrees extremely well with the experiments on HOPG; it is important to note that in the SAM simulations for graphite we use, according to the literature, as inplane Young's modulus $E_{\|}=1.046 \mathrm{TPa}$, and as $z$-axis (perpendicular to the planes) Young's modulus $E_{\perp}=(36.4 \pm 1) \mathrm{GPa}$ (more details are given in the Methods). Interestingly, when the Hertz model in (2) is applied to fit the experimental indentation curves measured on graphite, as if graphite was an isotropic material, the result of the fitting procedure gives as the single isotropic modulus $E_{\mathrm{Hertz}}^{\mathrm{HOPG}}=(33 \pm 3) \mathrm{GPa}$, for $R=100 \mathrm{~nm}$ like the AFM tip radius. The Hertz model fitting curve is also reported in Fig. 2a to show the perfect agreement with experiments and SAM simulations. The Hertz model is therefore able with a simple fitting procedure to obtain a value of Young's modulus that is equal, within an error of $10 \%$, to the most accepted value for the perpendicularto-the-plane Young's modulus of graphite, that is, $E_{\perp}=36 \mathrm{GPa}$.
The excellent consistency between experiment, simulation and Hertz model when studying the indentation of 2D films with extremely small indentation depths is a direct consequence of the following SAM observations. If we consider sub-interlayer distance indentations in a transversally isotropic (orthotropic) material having $E_{\perp}=E_{\text {Hertz }}$ and $E_{\|}$varying up to one order of magnitude compared with $E_{\text {Hertz }}$, we find that the contact pressure and contact area for a given pressure remain almost the same as in an isotropic material having $E=E_{\text {Hertz. }}$ On the other hand, the contact pressure changes markedly compared with the isotropic case when varying $E$ in the same range and maintaining $E_{\|}=E_{\text {Hertz }}$ (ref. 44). Overall these results indicate that for sub-nanometre AFM indentations, much smaller than the film's thickness, the force versus indentation curves are very sensitive to $E_{\perp}$, and almost independent of the value of $E_{\|}$. This result can be understood with a back-of-the-envelope calculation of the in-plane and perpendicular-to-the-plane stress distribution in a layered material when indenting the material perpendicular to the planes (for details see Supplementary Information and Supplementary Fig. 8). From these calculations it seems clear that the key parameter controlling sub-nanometre indentations is the ratio $a^{2} / l_{0} z_{\text {indent }}$, where $a$ is the contact radius, and $l_{0}$ is the length of an $s p^{2}$ bond. For example, when indenting graphite with an AFM tip with $z_{\text {indent }}<0.3 \mathrm{~nm}$, this simple model shows that we are sensing only $E_{\perp}$. A more precise analysis can be developed by using SAM calculations. In particular, the contact pressure distribution profiles in the case of sub-nanometre indentations for the Hertz model in the case of an isotropic material with $E_{\text {Hertz }}=33 \mathrm{GPa}$ (the value of $E_{\perp}$ in graphite), and in the case of $E_{\mathrm{Hertz}}=1.046 \mathrm{TPa}$ (the value of $E_{\|}$in graphite), are plotted in Fig. 2b along with the SAM simulations for bulk graphite and 


\section{Table 1. Summary of the experimental results of $E_{\perp}(\mathrm{GPa})$ at different RHs.}

\begin{tabular}{llllll} 
Relative humidity & $\mathbf{1 0} \pm \mathbf{2 \%}$ & $\mathbf{1 5} \pm \mathbf{3} \%$ & $\mathbf{2 5} \pm \mathbf{3} \%$ & $\mathbf{3 5} \pm \mathbf{3} \%$ & $\mathbf{5 0} \pm \mathbf{3} \%$ \\
\hline 10-layer EGO & $22 \pm 3$ & - & $23 \pm 4$ & $19 \pm 3$ & $22 \pm 3$ \\
Conventional GO & $21 \pm 6$ & $26 \pm 6$ & $35 \pm 10$ & - & $23 \pm 7$ \\
10-layer EG & - & - & $36 \pm 3$ & - & - \\
HOPG & - & - & $33 \pm 3$ & - & -
\end{tabular}

50-nm-thick graphite. It can be easily concluded that the pressure distributions for an isotropic material having $E=33 \mathrm{GPa}$, bulk graphite, and 50-nm-thick graphite are almost all the same. On the other hand, the pressure distribution changes markedly when

considering an isotropic material with $E=1.046 \mathrm{TPa}$. We conclude that when studying $2 \mathrm{D}$ materials with sub-nanometre indentations, the Hertz model is an extremely simple and accurate model to fit the experimental AFM indentation curves and obtain the perpendicular Young's modulus $E_{\perp}$ of $2 \mathrm{D}$ films having a thickness larger than the indentation depth.

\section{Perpendicular-to-the-plane elasticity}

After having tested and gathered a better understanding of subnanometre indentations in orthotropic layered materials such as bulk graphite, we have then used MoNI to investigate the interlayer elasticity of very thin films of layered materials. Initially, MoNI is performed on a film of 10-layer-thick EG grown on $\mathrm{SiC}$, as shown in Fig. 2c. We remark that $0.1 \mathrm{~nm}$ indentations in supported graphene films with a thickness of $\sim 10$ layers are not very sensitive to the presence of the $\mathrm{SiC}$ substrate, as also shown by SAM calculations. Similarly to the fitting procedure used in Fig. 2a, we use the Hertz model to fit the indentations curves on 10-layer EG. We have repeated several measurements, and the Hertz fit provides for 10-layer EG a modulus perpendicular to the planes equal to $E_{\perp}=(36 \pm 3) \mathrm{GPa}$, the same value as ideal graphite, and larger than the value measured on $\mathrm{HOPG}\left(E_{\perp}=33 \pm 3 \mathrm{GPa}\right)$. It is worth noting that in Fig. 2a for the SAM simulations and the MoNI experiments on HOPG we use a tip radius of $R=(100 \pm 10) \mathrm{nm}$, whereas the MoNI experiment on 10-layer EG in Fig. 2c was performed with an AFM tip of $R=(114 \pm 10) \mathrm{nm}$; therefore, to compare these curves we need to rescale them by a factor of $R^{1 / 2}$ (see equation (2)).

To investigate the role of the substrate and number of layers, we have then focused our experiments on the first layers of EG grown on the $\mathrm{Si}$ face of $\mathrm{SiC}(0001)$. More details on these experiments are reported in the Methods, in the Supplementary Information and in Supplementary Fig. 7. Much attention in the scientific community is at present directed towards single-layer EG grown in the Si face of $\mathrm{SiC}$ (refs 31,47). This system is very interesting because between the $\mathrm{SiC}$ substrate and the first 'well-decoupled' 1-layer graphene, there is a honeycomb carbon layer called the buffer layer ${ }^{31}$ that has different properties from graphene, and it has a strong interaction with the $\mathrm{SiC}$ substrate. This substrate interaction strongly modifies the electronic structure of the carbon buffer layer, which is in fact not conducting. Extensive research is underway about the properties of the decoupled first 1-layer graphene and the buffer layer on SiC. Here, we have performed MoNI experiments on the decoupled first 1-layer graphene and on the buffer layer grown on the $\mathrm{Si}$ face of $\mathrm{SiC}$, as shown in Fig. $2 \mathrm{c}$ and Supplementary Fig. 7. We find that the buffer layer produces indentation curves very similar to the ones on bare $\mathrm{SiC}$ (also reported in Fig. 2c). This result is in agreement with the notion that $\mathrm{C}$ atoms in the buffer layer have a strong interaction with the $\mathrm{SiC}$ substrate; indeed this interaction is often described as covalent bonding ${ }^{31}$. On the other hand, we obtain stiffer indentation curves on the decoupled 1-layer graphene on $\mathrm{SiC}$. These curves are stiffer than those ones measured on the buffer layer, and on 10layer EG, as clear in Fig. 2c and Supplementary Fig. 7. This result can be understood by the following considerations. For a film of 10-layer graphene, we are probing only $E_{\perp}$, which is $\sim 36 \mathrm{GPa}$, because the $0.1 \mathrm{~nm}$ indentation is easily distributed among the 0.01 $\mathrm{nm}$ displacements per layer, supposing an infinitely rigid substrate. In contrast, when the AFM tip is indenting $\left(z_{\text {indent }} \sim 0.1 \mathrm{~nm}\right) 1$ layer EG on an infinitely rigid substrate, the whole indentation is carried on by that single layer sitting at an interatomic distance of $0.3 \mathrm{~nm}$ from the substrate, and strongly resisting such a huge deformation. Therefore, for 1-layer graphene on $\mathrm{SiC}$ we are now probing a complex convolution of effects and properties, which include $E_{\|}(\sim 1 \mathrm{TPa}), E_{\perp}$, and the elasticity of the $\mathrm{SiC}$ substrate $(E \sim$ $400 \mathrm{GPa}$ ). In conclusion, these experiments show that MoNI measurements can be sensitive to the substrate interaction and to the number of layers, although more calculations will be required to fully explain the results for 1-layer graphene.

The ability of the Hertz relationship in equation (2) to model sub-nanometre indentations in transversally isotropic 2D materials is further confirmed by Fig. 2d. We have fitted all (except for 1-layer EG) the measured $F_{z}\left(z_{\text {indent }}\right)$ curves with Hertz equation (2) leaving as a free fitting parameter the exponent, $b$, of $z_{\text {indent, }}$ that is, by using the function $F_{z}=C \cdot z_{\text {indent }}^{b}$, where $C$ is a constant. The results are reported in Fig. 2 d, and they show that $b$ is on average equal to $(1.4 \pm 0.1)$, a value in very close agreement with the exponent expected for a perfect Hertzian contact where $b=1.5$ (see equation (2)). By fitting with the Hertz equation (2) all of the exper-imental indentation curves for the different 2D materials films in ambient atmosphere (see Table 1), we find that as mentioned above the perpendicular elastic modulus is larger in 10layer EG films than in HOPG; this difference is probably related to the absence of defects in 10-layer EG compared with HOPG. We also find that 10-layer EGO is much softer than 10-layer EG; this result can be explained by the increase of the interlayer distance $d$, in EGO compared with EG, precisely from $d=3.4 \AA$ to $d=9.3 \AA$ (ref. 48) as also ob-served in X-ray diffraction spectroscopy measurements ${ }^{49}$. The more surprising result is the difference in the perpendicular elasticity between EGO films and GO films produced with the conventional exfoliation/filtration/deposition method (see Methods). In ambient humidity we precisely obtain: $E_{\perp}=(35 \pm 10) \mathrm{GPa}$ (for conventional GO), $E_{\perp}=(36 \pm 3) \mathrm{GPa}$ (for 10 -layer EG), $E_{\perp}=(33 \pm 3) \mathrm{GPa}$ (for HOPG), and $E_{\perp}=(23 \pm 4)$ $\mathrm{GPa}$ (for EGO). To understand the origin of the large interlayer perpendicular elasticity in conventional GO compared with EGO, we have performed DFT calculations on the elasticity of GO with a different amount of intercalated water. We have then compared these calculations with MoNI experiments on GO and EGO performed at different ambient humidities.

\section{Density functional theory study of perpendicular elasticity} DFT calculations have been performed on model structures of GO consisting of periodic stacks of graphene layers fully oxidized by either hydroxyl or epoxide groups, including increasing concentrations of water molecules, and presenting AA stacking (see Supplementary Information and Table 1). For each model, we used a DFT-D2 (refs 49,50) scheme (technical details and the full list of calculations are discussed in Supplementary Information, and Supplementary Figs 9-12) to perform a full structural optimization and determine the zero-temperature interlayer spacing. Subsequently, we applied a pressure and used DFTD2 to estimate the $z$-axis Young's modulus from the energy versus displacement curves. A summary of the DFT results reporting $E$ and interlayer distance as a function of intercalated water percentage for GO structures fully oxidized with hydroxyls is reported in Table 2. Figure $3 \mathrm{a}$ shows the calculated $F_{z}$ versus displacement curves at varying intercalated water content for the case of graphene fully oxidized with hydroxyl groups, because 
a

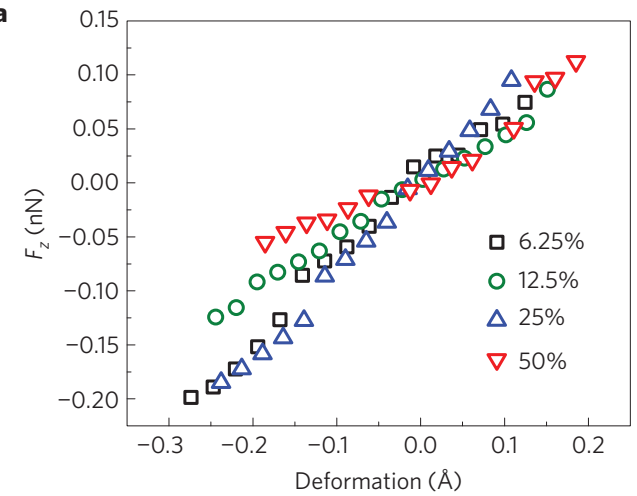

b

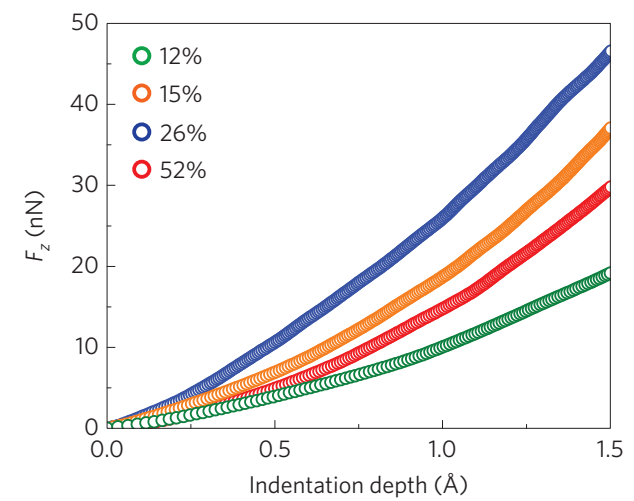

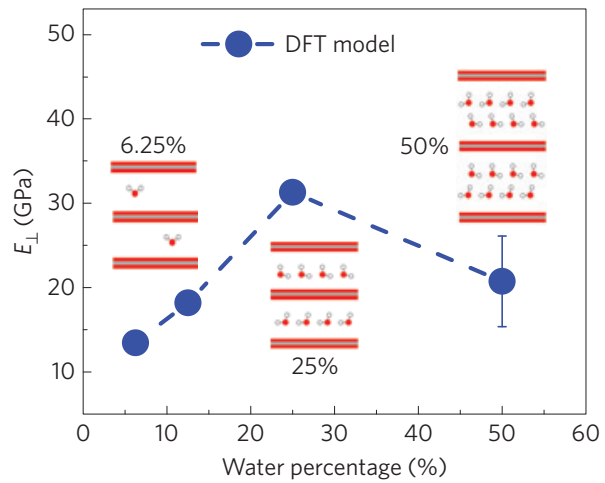

d

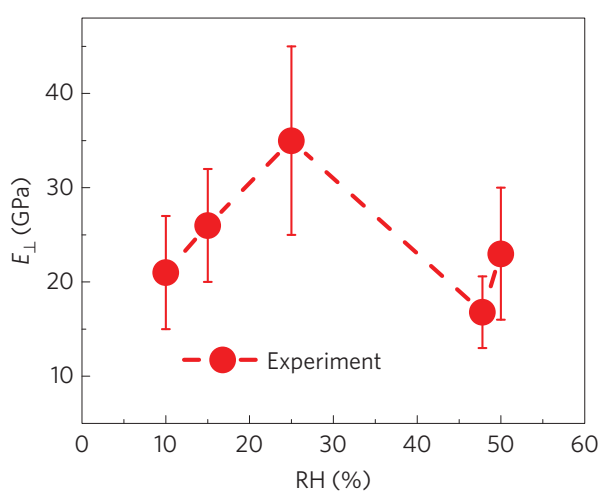

Figure 3. DFT and experimental results for conventional GO films. a, The DFT-calculated $F_{z}$ versus displacement curves for different water contents in graphene fully oxidized with hydroxyl groups. b. Experimental $F_{z}$ versus indentation depth curves at different RHs in conventional GO. All of the curves were obtained with the same AFM tip. c,d, Experimental and DFT results of $E_{\perp}$ of GO as a function of water content and RH, respectively. The insets are

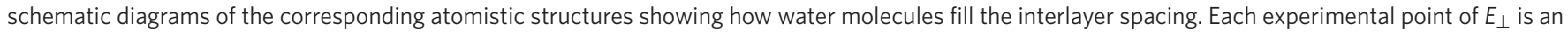
average value of more than 30 different measurements.

previous experiments have shown that conventional GO films are mainly composed of hydroxyls groups ${ }^{36}$. In comparison, Fig. $3 \mathrm{~b}$ presents the experimental MoNI results on conventional GO at different relative humidities (RHs). Figure $3 \mathrm{c}$,d shows the resulting $E_{\perp}$ as a function of intercalated water percentage compared with carbon (for DFT calculations) and RH (for MoNI experiments), respectively. A summary of the experimental results is also reported in Table 1 . The agreement between experiments and DFT calculations is striking. DFT calculations and experiments show that $E_{\perp}$ increases with the amount of intercalated $\mathrm{H}_{2} \mathrm{O}$ molecules (and $\mathrm{RH}$ ), reaching a maximum value of about $31 \mathrm{GPa}$ at $25 \% \mathrm{H}_{2} \mathrm{O}$, and $35 \mathrm{GPa}$ at $\mathrm{RH}=25 \%$, for the DFT calculations and indentation experiments, respectively. The perpendicular elastic modulus then decreases down to $20 \mathrm{GPa}$ at $50 \%$ water, and $23 \mathrm{GPa}$ at $50 \% \mathrm{RH}$, for the DFT calculations and indentation experiments, respectively. We remark that the presence of a maximum in the perpendicular modulus found in both experiments and calculations is a key result; however, the excellent quantitative agreement between water content (from DFT) and $\mathrm{RH}$ (from experiments) might be only a coincidence. The DFT calculations clearly give an insight into the atomistic origin of the behaviour of $E_{\perp}$ as a function of intercalated water. DFT calculations show that the interlayer distance and perpendicular Young's modulus in GO change abruptly between the case of dry layers and a multilayer film including a small amount of $\mathrm{H}_{2} \mathrm{O}(<6 \%)$, whereas more gradual variations of these physical properties are obtained for increasing the water content between $6.25 \%$ and $25 \%$ (Fig. 3c, and Table 2). In particular, $E_{\perp}$ drops from about $35 \mathrm{GPa}$ (close to EG) in dry films to about 11-14 GPa when the layered structure includes $6.25 \% \mathrm{H}_{2} \mathrm{O}$. This behaviour can be understood by considering that when the amount of water is only a few percentage of the carbon amount, $\mathrm{H}_{2} \mathrm{O}$ molecules swell the graphene structure, increasing the interlayer distance from $3.4 \AA$ to about $6.2 \AA$, but leaving the interlayer space mainly empty and therefore producing a soft structure with a low perpendicular elastic modulus. This interlayer modulus increases with increasing amount of water, which fills the interlayer space without changing too much the interlayer distance (Table 2). However, at 25\% water, $\mathrm{H}_{2} \mathrm{O}$ molecules have completely filled a water layer in between the layers, and this situation corresponds with the maximum in perpendicular elastic modulus. Above $25 \%$ water, the perpendicular elastic modulus decreases because a second water layer starts to form in between the layers, further swelling and softening the GO structure. Owing to this explanation, it is now possible to understand the different values of $E_{\perp}$ in EGO compared with conventional GO. EGO is not a porous structure, and water intercalation is minimal ${ }^{49}$ and independent of humidity. For this reason we find that $E_{\perp}$ in $\mathrm{EGO}$ remains constant and $\sim 22 \mathrm{GPa}$ for all RHs. On the other hand, conventional GO is a porous structure ${ }^{3}$ where the amount of intercalated water can change depending on the humidity; therefore, in agreement with the DFT calculations we observe a maximum in conventional GO when varying the $\mathrm{RH}$.

\section{Outlook}

In conclusion, we have presented a new methodology, which combines sub-ångström-resolution indentation measurements and SAMs to study the elasticity perpendicular to the plane of few-layerthick 2D materials. The comparison between indentation experiments and SAMs has also demonstrated that a simpler approach to interpret experimental sub-nanometre indentation curves in fewlayer-thick 2D films is to use the Hertz model. We showed that the fitting of the experimental indentation curves with the Hertz model provides, to a good approximation, the value of Young's modulus 


\section{Table 2. Summary of the DFT results reporting interlayer distance and $E_{\perp}$ as a function of different fractions of intercalated water for GO structures fully oxidized with hydroxyls.}

\begin{tabular}{lll} 
Water fraction (\%) & Interlayer distance $(\AA)$ & $\boldsymbol{E}_{\perp}(\mathrm{GPa})$ \\
\hline 0 & 6.2 & 34.9 \\
6.25 & 8.6 & 13.4 \\
12.5 & 8.7 & 18.2 \\
25 & 8.9 & 31.3 \\
50 & 11.3 & 20.7
\end{tabular}

perpendicular to the plane of $2 \mathrm{D}$ films. The experimental study of EG and different types of GO film, combined with DFT calculations, has demonstrated that the interlayer elasticity is extremely sensitive to the presence of intercalated molecules in between the planes. In particular, these studies show that intercalated water in GO can markedly change the interlayer elastic modulus, which at first decreases when a small amount of water is intercalated in between the layers and the structure is swelled, and then increases with increasing amount of water until $\mathrm{H}_{2} \mathrm{O}$ molecules have completely filled a water layer in between the layers. Above this point, the perpendicular elastic modulus decreases because a second water layer starts to form in between the layers, further swelling and softening the GO structure. This understanding can also explain the different behaviour of $E_{\perp}$ in conventional GO and EGO. Finally, the here proposed subångström-resolution indentation measurements applied to EG are shown to be sensitive to the substrate interaction and to the number of layers.

The results reported here provide a new path to study the interlayer elastic coupling and the Van der Waals forces in few-layerthick 2D materials, and shed new light on the use of the Hertz model in investigating the perpendicular-to-the-plane Young's modulus of $2 \mathrm{D}$ films. This study will impact a variety of fields, from electronics to phononics, allowing new investigations and understanding of the relationship between molecular structure, thermal conductivity, electronic properties, and phonon propagation in layered materials. For example, through local measurements of the elastic modulus, MoNI could probe interlayer and substrate interaction, as well as the presence of dopants/intercalates, which are extremely important for modulating the electronic properties of $2 \mathrm{D}$ materials. Furthermore, the interlayer elasticity is strictly connected with the out-ofplane thermal properties of layered materials. The here discussed interlayer elasticity measurements could help in the understanding of the origin of the extremely low out-of-plane thermal conductivity found in thin films of transition metal dichalcogenides ${ }^{30}$.

\section{References}

1. Park, J. Y., Kwon, S. \& Kim, J. H. Nanomechanical and charge transport properties of two-dimensional atomic sheets. Adv. Mater. Interfaces $\mathbf{1}$, 130089 (2014).

2. Berger, C. et al. Electronic confinement and coherence in patterned epitaxial graphene. Science 312, 1191-1196 (2006).

3. Zhu, Y. et al. Microwave assisted exfoliation and reduction of graphite oxide for ultracapacitors. Carbon 48, 2118-2122 (2010).

4. Lin, Y-M. et al. Operation of graphene transistors at gigahertz frequencies. Nano Lett. 9, 422-426 (2008).
5. Wang, X., Zhi, L. \& Müllen, K. Transparent, conductive graphene electrodes for dye-sensitized solar cells. Nano Lett. 8, 323-327 (2008).

6. Yu, W. J. et al. Vertically stacked multi-heterostructures of layered materials for logic transistors and complementary inverters. Nature Mater. 12, 246-252 (2013)

7. Pesin, D. \& MacDonald, A. H. Spintronics and pseudospintronics in graphene and topological insulators. Nature Mater. 11, 409-416 (2012).

8. Novoselov, K. S. et al. Electric field effect in atomically thin carbon films. Science 306, 666-669 (2004).

9. De Heer, W. A. et al. Epitaxial graphene. Solid State Commun. 143, 92-100 (2007)

10. Lee, C., Wei, X., Kysar, J. W. \& Hone, J. Measurement of the elastic properties and intrinsic strength of monolayer graphene. Science 321, 385-388 (2008).

11. Baringhaus, J. et al. Exceptional ballistic transport in epitaxial graphene nanoribbons. Nature 506, 349-354 (2014).

12. Bolotin, K. I. et al. Ultrahigh electron mobility in suspended graphene. Solid State Commun. 146, 351-355 (2008).

13. Balandin, A. A. et al. Superior thermal conductivity of single-layer graphene. Nano Lett. 8, 902-907 (2008)

14. Chiu, H. C., Kim, S., Klinke, C. \& Riedo, E. Morphology dependence of radial elasticity in multiwalled boron nitride nanotubes. Appl. Phys. Lett. 101, 103109 (2012)

15. Loh, K. P., Bao, Q., Eda, G. \& Chhowalla, M. Graphene oxide as a chemically tunable platform for optical applications. Nature Chem. 2, 1015-1024 (2010)

16. Eda, G., Fanchini, G. \& Chhowalla, M. Large-area ultrathin films of reduced graphene oxide as a transparent and flexible electronic material. Nature Nanotech. 3, 270-274 (2008)

17. Wei, Z. et al. Nanoscale tunable reduction of graphene oxide for graphene electronics. Science 328, 1373-1376 (2010).

18. Ci, L. et al. Atomic layers of hybridized boron nitride and graphene domains. Nature Mater. 9, 430-435 (2010).

19. Ramakrishna Matte, H. S. S. et al. $\mathrm{MoS}_{2}$ and $\mathrm{WS}_{2}$ analogues of graphene. Angew. Chem. 122, 4153-4156 (2010).

20. Castellanos-Gomez, A. et al. Mechanical properties of freely suspended semiconducting graphene-like layers based on $\mathrm{MoS}_{2}$. Nanoscale Res. Lett. 7, 1-4 (2012).

21. Radisavljevic, B., Radenovic, A., Brivio, J., Giacometti, V. \& Kis, A. Single-layer $\mathrm{MoS}_{2}$ transistors. Nature Nanotech. 6, 147-150 (2011)

22. Kelly, B. T. Physics of Graphite (Applied Science, 1981).

23. Kwon, S. et al. Probing nanoscale conductance of monolayer graphene under pressure. Appl. Phys. Lett. 99, 013110 (2011).

24. Lee, C. et al. Frictional characteristics of atomically thin sheets. Science 328, 76-80 (2010).

25. Lee, C. et al. Elastic and frictional properties of graphene. Phys. Status Solidi B 246, 2562-2567 (2009).

26. Stewart, J. A. \& Spearot, D. E. Atomistic simulations of nanoindentation on the basal plane of crystalline molybdenum disulfide $\left(\mathrm{MoS}_{2}\right)$. Modelling Simul. Mater. Sci. Eng. 21, 045003 (2013).

27. Suk, J. W., Piner, R. D., An, J. \& Ruoff, R. S. Mechanical properties of monolayer graphene oxide. ACS Nano 4, 6557-6564 (2010).

28. Hajgató, B. et al. Out-of-plane shear and out-of plane Young's modulus of double-layer graphene. Chem. Phys. Lett. 564, 37-40 (2013)

29. Nakamura, N., Ogi, H. \& Hirao, M. Resonance ultrasound spectroscopy with laser-Doppler interferometry for studying elastic properties of thin films. Ultrasonics 42, 491-494 (2004)

30. Chiritescu, C. et al. Ultralow thermal conductivity in disordered, layered WSe crystals. Science 315, 351-353 (2007).

31. Riedl, C., Coletti, C. \& Starke, U. Structural and electronic properties of epitaxial graphene on $\operatorname{SiC}(000$ 1): A review of growth, characterization, transfer doping and hydrogen intercalation. J. Phys. D 43, 374009 (2010).

32. Lucas, M. et al. Hindered rolling and friction anisotropy in supported carbon nanotubes. Nature Mater. 8, 876-881 (2009).

33. Tan, P. H. et al. The shear mode of multilayer graphene. Nature Mater. 11, 294-300 (2012)

34. Lucas, M., Mai, W., Yang, R., Wang, Z. L. \& Riedo, E. Aspect ratio dependence of the elastic properties of $\mathrm{ZnO}$ nanobelts. Nano Lett. 7, 1314-1317 (2007).

35. Palaci, I. et al. Radial elasticity of multiwalled carbon nanotubes. Phys. Rev. Lett. 94, 175502 (2005).

36. Kim, S. et al. Room-temperature metastability of multilayer graphene oxide films. Nature Mater. 11, 544-549 (2012).

37. Hummers, W. S. Jr \& Offeman, R. E. Preparation of graphitic oxide. J. Am. Chem. Soc. 80, 1339-1339 (1958).

38. Lantz, M., O'Shea, S., Welland, M. \& Johnson, K. Atomic-force-microscope study of contact area and friction on $\mathrm{NbSe}_{2}$. Phys. Rev. B $\mathbf{5 5}$, 10776-10785 (1997). 
39. Carpick, R. W., Ogletree, D. \& Salmeron, M. Lateral stiffness: A new nanomechanical measurement for the determination of shear strengths with friction force microscopy. Appl. Phys. Lett. 70, 1548-1550 (1997).

40. Grierson, D., Flater, E. \& Carpick, R. Accounting for the JKR-DMT transition in adhesion and friction measurements with atomic force microscopy. J. Adhes. Sci. Technol. 19, 291-311 (2005).

41. Derjaguin, B. V., Muller, V. M. \& Toporov, Y. P. Effect of contact deformations on the adhesion of particles. J. Colloid Interface Sci. 53, 314-326 (1975).

42. Johnson, K. L. Contact Mechanics (Cambridge Univ. Press, 1987).

43. Turner, J. Contact on a transversely isotropic half-space, or between two transversely isotropic bodies. Int. J. Solids Struct. 16, 409-419 (1980).

44. Bagault, C., Nelias, D., Baietto, M-C. \& Ovaert, T. C. Contact analyses for anisotropic half-space coated with an anisotropic layer: Effect of the anisotropy on the pressure distribution and contact area. Int. J. Solids Struct. 50, 743-754 (2013).

45. Bagault, C., Nelias, D. \& Baietto, M-C. Contact analyses for anisotropic half space: Effect of the anisotropy on the pressure distribution and contact area. J. Tribol. 134, 031401 (2012).

46. Jacq, C., Nelias, D., Lormand, G. \& Girodin, D. Development of a three-dimensional semi-analytical elastic-plastic contact code. J. Tribol. 124, 653-667 (2002).

47. Palacio, I. et al. Atomic structure of epitaxial graphene sidewall nanoribbons: Flat graphene, miniribbons, and the confinement gap. Nano Lett. 15, 182-189 (2014).

48. Zhou, S. \& Bongiorno, A. Origin of the chemical and kinetic stability of graphene oxide. Sci. Rep. 3, 2484 (2013).

49. Zhou, S. et al. Film structure of epitaxial graphene oxide on SiC: Insight on the relationship between interlayer spacing, water content, and intralayer structure. Adv. Mater. Interface 1, 1300106 (2014).
50. Giannozzi, P. et al. QUANTUM ESPRESSO: A modular and open-source software project for quantum simulations of materials. J. Phys. Condens. Matter 21, 395502 (2009).

\section{Acknowledgements}

Y.G., S.K., H-C.C. and E.R., acknowledge the support of the Office of Basic Energy Sciences of the US Department of Energy (DE-FG02-06ER46293). S.Z. and A.B. acknowledge the support of the National Science Foundation (NSF) grant CMMI 1436375. S.Z., A.B., C.B. and W.d.H. acknowledge the support of the NSF grant DMR-0820382. C.B. acknowledges partial financial support from the European Flagship Graphene. A.B. acknowledges the support of the NSF grant CHE-0946869. R.S. acknowledges the support of the Italian Cariplo Foundation, project No. 2011-0373. We thank J-P. Turmaud for the EG on Si sample. 


\section{Methods}

Modulated nanoindentation measurements. The oscillations are applied at $1 \mathrm{kHz}$ to the atomic force microscopy (AFM) tip by a piezoelectric stage rigidly attached to the AFM cantilever-tip system, and controlled by a lock-in amplifier (Stanford Research Systems, SR830), while a constant normal force $F_{z}$ between the tip and the 2D films is maintained constant by the feedback loop of the AFM (see Supplementary Information for more details). To maintain the linear elastic regime, the piezo-stage oscillations are chosen to be only $\sim 0.1 \AA$. During the indentation, the driving fixed piezo-stage oscillation amplitude $\Delta z_{\text {piezo }}$ is equal to the sum of the cantilever bending and tip-2D film normal deformation. Under such circumstances, the AFM cantilever and the tip-film contact can be considered as two springs connected in series: the cantilever with stiffness $k_{\mathrm{lev}}$ and the tip-film contact with stiffness $k_{\text {cont. }}$. The force required to stretch these two springs in series with a total displacement $\Delta z_{\text {piezo }}$ is equal to the normal force variation $\Delta F_{z}$. This experimental configuration allows us to measure the total stiffness $k_{\text {tot }}$ at each normal load $F_{z}$, fixed by the feedback loop of the AFM, from the following relation: $\left(\Delta F_{z} / \Delta z_{\text {piezo }}\right)=k_{\text {tot }}\left(F_{z}\right)\left(\left(1 / k_{\text {lev }}\right)+\left(1 / k_{\text {cont }}\right)\right)^{-1}$ where $\Delta F_{z}$ is the variation of the normal force caused by the piezo-stage oscillation and is much smaller than $F_{z}, k_{\mathrm{lev}}$ is the spring constant of the cantilever, and $k_{\text {cont }}$ is the tip-film contact stiffness. As $k_{\text {lev }}$ can be measured independently, the measurement of $\Delta F_{z} / \Delta z_{\text {piezo }}$ at different normal loads $F_{z}$ allows us to acquire the tip-film contact stiffness $k_{\text {cont }}$ as a function of $F_{z}$. Force versus indentation curves are obtained by integrating

$\mathrm{d} F_{z}=k_{\text {cont }}\left(F_{z}\right) \cdot \mathrm{d} z_{\text {indent }}$. The data reported in Table 1 are averaged values obtained from 10, 210, 90 and 12 measurements on highly oriented pyrolytic graphite, graphene oxide (GO), epitaxial graphene oxide (EGO) and epitaxial graphene (EG) respectively. More details on the MoNI experiments are reported in the Supplementary Information.

2D materials. We have studied 10-layer graphene grown epitaxially on the $\mathrm{C}$ face of a $4 \mathrm{H}-\mathrm{SiC}$ substrate ${ }^{2}$. We have also investigated the buffer layer and the first decoupled 1-layer graphene, both grown epitaxially on the Si face of 4H-SiC (0001) (refs 31,47; see Supplementary Fig. 7).

To prepare EGO samples, the 10-layer EG samples on $\mathrm{SiC}$ chips are then directly oxidized shortly after preparation by using a milder Hummers' method ${ }^{49}$, which avoids graphene exfoliation and dispersion in solution. Once the reactions are terminated, EGO films on the $\mathrm{SiC}$ chips are picked up from the solution and rinsed with deionized water for $1 \mathrm{~min}$. The EGO films, 10 layers thick, are finally blow-dried by nitrogen gas.

GO films are prepared by drop-casting colloidal GO dispersion on a Si chip and leaving it to dry at $80^{\circ} \mathrm{C}$. Stable colloidal GO dispersions are produced by a modified Hummers' method ${ }^{37}$. Once the reaction is terminated, the oxidized portion is separated and cleaned from unoxidized graphite and other residual species. The cleaning is performed by centrifugation of the obtained suspension at $7,197 \mathrm{~g}$ for $30 \mathrm{~min}$ to remove both the acidic content and ions. The solid content is collected and redispersed with deionized $\mathrm{H}_{2} \mathrm{O}$. This operation is repeated in sequence until the $\mathrm{pH}$ of the supernatant is close to neutrality. At that point, the exfoliation of graphite oxide is performed by prolonged and vigorous shaking, forming a brownish colloidal suspension of GO flakes. The subsequent collection of the purified supernatant results in stable aqueous GO suspensions, which are drop-casted on Si to form a film with a thickness of 40-50 nm (ref. 51).

Semi-analytical method. The theoretical model used here to predict the indentation curves as well as the distribution of contact pressure and the contact area is based on what is known as semi-analytical methods. SAM is an alternative to the finite element method, quite close to the boundary element method, suitable for continuum mechanics problems. The main advantage of the boundary element method over SAM is that the first one is more versatile because it uses surface or volume integrals whatever the shape of the volume of interest. Conversely, SAM uses analytical solutions of these integrals for simple geometries (such as sphere or cuboid), which reduces the computation to a simple summation of elementary solutions. SAM consists of the numerical summation of elementary analytical solutions, such as the effect of a point load on a layered half-space, as far as they are known. The difficulty for anisotropic elastic coating and/or substrate is that the set of equations to be solved requires manipulating complex numbers with conjugate pairs, leading to double roots when the material tends to behave as elastically isotropic. One of the advantages of SAMs when applied to the contact problem compared with more widely used numerical techniques is the computing time, which is at least one or two orders of magnitude shorter. For more details on SAM for the numerical procedure used to solve the contact problem for an elastic substrate coated with an anisotropic layer, see refs 44,45 .

For transversely isotropic material, there are 5 independent elastic constants in the elasticity stiffness tensor: $C_{11}, C_{12}, C_{13}, C_{33}$ and $C_{44}$. These 5 elastic constants can be transformed to the well-known engineering notation in the following way.

$$
\begin{gathered}
E_{\|}=E_{x}=E_{y}=\left(C_{11}-C_{12}\right)\left(C_{11} C_{33}+C_{12} C_{33}-2 C_{13} C_{13}\right) / \\
\left(C_{11} C_{33}-C_{13} C_{13}\right) \\
E_{\perp}=E_{z}=C_{33}-2 C_{13} C_{13} /\left(C_{11}+C_{12}\right) \\
v_{z x}=C_{13} /\left(C_{11}+C_{12}\right) \\
G_{x z}=C_{44} \\
G_{x y}=\left(C_{11}-C_{12}\right) / 2 \quad v_{x y}=v_{y x}=\left(E_{x} / G_{x y}\right)-1
\end{gathered}
$$

Note that $G_{x y}$ and $v_{x y}$ are correlated, which means that only one of them is independent. Graphite's elastic constants used for SAM have been previously reported in the literature ${ }^{22}$ and they are $C_{11}=(1,060 \pm 20) \mathrm{GPa}$,

$C_{12}=(180 \pm 20) \mathrm{GPa}, C_{13}=(15 \pm 5) \mathrm{GPa}, C_{33}=(36.5 \pm 1) \mathrm{GPa}$ and

$C_{44}=(4.5 \pm 0.5) \mathrm{GPa}$. For graphite, this gives $E_{\|}=(1.06 \pm 0.02) \mathrm{TPa}$, and $E_{\perp}=(36.4 \pm 1) \mathrm{GPa}$.

The $\mathrm{SiC}$ substrate was approximated as cubic with $E=450 \mathrm{GPa}, \nu=0.17$ and $G=E / 2(1+v)=192.31 \mathrm{GPa}$.

DFT calculation. Density functional theory (DFT) calculations are performed by using the PWscf code of the QUANTUM Espresso toolkit ${ }^{50}$. We use a plane-wave basis set with an energy cutoff of 120 Ry to represent the Kohn-Sham wavefunction, norm-conserving pseudo-potentials for all atomic species ${ }^{52}$, and the exchange-correlation functional proposed by Perdew, Burke and Ernzerhof ${ }^{53}$. To model hydrated GO, we consider two ordered structures-graphene sheets fully covered by either hydroxyl or epoxide groups, with various amounts of water molecules intercalated in between the oxide layers ${ }^{36,48,49}$. The GO sheets are arranged in either 'AA' or 'AB' stacking geometry. We use the primitive unit cell of each model as the supercell. We use $3 \times 3 \times 3$ and $3 \times 3 \times 1$ gamma-centred Monkhorst-Pack meshes to sample the Brillouin zone of the supercells including one and two oxide layers, respectively. To account for the London dispersion forces in multilayer GO, we adopt the semi-empirical DFT-D2 approach proposed previously ${ }^{54}$. The GO models are fully optimized for both the electronic and cell degrees of freedom using the DFT-D2 scheme. To calculate the perpendicular-to-the-plane Young's modulus, we started from the equilibrium structure of each model, and we vary the lattice parameter along the $z$ direction with a strain step of $\sim 0.2 \%$ up to a $2 \%$ strain. The ionic positions of GO models are then gently relaxed to obtain the total energy and stress tensor under each strain. The $E_{\perp}$ value of each model is calculated by interpolating the pressure along the $z$ direction with respect to the volume using the equation: $E_{\perp}=-V \mathrm{~d} P / \mathrm{d} V$, where $P$ is the pressure along the $z$ direction, and $V$ is the volume of GO models. More details are reported in the Supplementary Information.

\section{References}

51. Carroll, K. M. et al. Parallelization of thermochemical nanolithography. Nanoscale 6, 1299-1304 (2014).

52. Troullier, N. \& Martins, J. L. Efficient pseudopotentials for plane-wave calculations. Phys. Rev. B 43, 1993-2006 (1991).

53. Perdew, J. P., Burke, K. \& Ernzerhof, M. Generalized gradient approximation made simple. Phys. Rev. Lett. 77, 3865-3868 (1996).

54. Grimme, S. Semiempirical GGA-type density functional constructed with a long-range dispersion correction. J. Comput. Chem. 27, 1787-1799 (2006). 